\title{
Encephalomyelitis with seroconversion to Listeria
} monocytogenes

\author{
P Baxter, D Gardner-Medwin, D Bates, L Ragunathan, H R Ingham
}

\begin{abstract}
A previously healthy girl recovered from a lymphocytic encephalomyelitis with brain stem involvement after antibiotic and steroid treatment. Cultures of blood and cerebrospinal fluid were negative, but she seroconverted to Listeria monocytogenes serotype 4. Antilisterial treatment should be considered in similar cases.
\end{abstract}

Acute meningoencephalitis with a scanty cerebrospinal fluid lymphocytosis, normal cerebrospinal fluid glucose and protein concentrations, and a normal computed tomogram is usually considered to be viral. In adult patients listerial infection is a recognised cause, especially if there is brain stem involvement, but this has not been described in children. ${ }^{1}$

\section{Case report}

A 10 year old girl with an unremarkable medical history presented to another hospital with a four day history of headache and malaise, which was treated as sinusitis for two days with oral amoxycillin. Fever, herpes labialis, neck stiffness, and mild confusion were noted, but no focal signs. Her cerebrospinal fluid had 22 lymphocytes $/ \mathrm{ml}$, with normal glucose and protein concentrations and no growth on culture. Over the next 24 hours she became dysarthric and incontinent. On transfer (day 1) she was unresponsive to command, with flexor withdrawal from pain, mild neck stiffness, early papilloedema, sluggish pupil responses to light, trismus, hyperreflexia in the arms, diminished tone in the legs, and generalised high voltage delta activity in her eletroencephalogram. Computed tomography showed no focal lesion and no evidence of raised pressure. She was treated with intravenous benzylpenicillin and chloramphenicol for five days and acyclovir for 10 days. Over the next two days she developed obligate doll's eye movements and a right abducens palsy. Repeat lumbar puncture showed pressure of $10.5 \mathrm{~cm} \mathrm{H}_{2} \mathrm{O}(1.03 \mathrm{kPa})$ and 67 lymphocytes $/ \mathrm{ml}$, with no growth. On day 4 she had a respiratory arrest, leading to assisted ventilation for two weeks. She also developed a complete flaccid paraplegia with urinary retention. At this point she was started on dexamethasone, which was continued for one week and then weaned over one week. On day 7 she opened her eyes spontaneously and moved her left hand to command. On day 10 she became feverish again and was treated with intravenous amoxycillin for Escherichia coli in her urine. A repeat computed tomogram was unchanged. Her conscious level and motor function gradually improved. On day 17 nerve conduction velocity and evoked amplitudes were normal but $\mathbf{F}$ responses were few or absent. Between days 22 and 28 she was lifting both arms to command and communicating by sign language, but had obligate doll's eye movements, brisk jaw jerk, bilateral facial palsy, palatal weakness, poor cough, a flaccid paraplegia, and absent pin prick sensation from T8 to T12. By day 40 she was communicating verbally with no evidence of intellectual deficit, could write legibly, but had appreciable emotional lability, mild left facial and palatal weakness, and an asymetrical mainly distal paraparesis and needed a frame to walk. By day 95 she only had minimal signs of a left hemiparesis.

Bacterial and viral culture of cerebrospinal fluid, blood, and stool were all negative. There were no change in blood and cerebrospinal fluid titres to herpes viruses, mycoplasma, or borrelia. Blood taken on days 1 and 9 showed a rise in titre to Listeria monocytogenes type 4 from $<16$ to 32 , which is regarded as seroconversion.

\section{Discussion}

This is the first case we can find associating $L$ monocytogenes infection in a child with encephalitis involving the brain stem, myelitis and possible radiculitis, suggested by the abnormal F responses. In children listerial infection of the nervous system usually causes meningitis. ${ }^{2}$ In adults a range of disease is reported, including meningitis, meningoencephalitis, which is distinguished by the presence of focal signs, and rhombencephalitis, where only the brain stem is affected. ${ }^{134}$ The meningitic form usually has a cerebrospinal fluid polymorphonuclear leucocytosis (approximately $2000 / \mathrm{ml}$ ), low glucose and high protein concentrations in the cerebrospinal fluid, and positive blood and cerebrospinal fluid cultures. The meningoencephalitic form has a lower, predominantly lymphocytic, cerebrospinal fluid cell count (approximately $200 / \mathrm{ml}$ ), frequently normal glucose and protein concentrations in the cerebrospinal fluid and the cerebrospinal fluid or blood is culture negative in $25 \%$ of cases. ${ }^{3}$ The rhombencephalitic form usually show only a scanty cerebrospinal fluid lymphocytosis and culture is usually negative, and the diagnosis is based on positive blood cultures, which can also be repeatedly negative in some patients. Reported cases of rhombencephalitis have a high mortality (30\%) and a characteristic postmortem feature is the presence of microabscesses. ${ }^{45}$ There is one case 
report of an unusual relapsing rhombencephalitis associated with seroconversion to $L$ monocytogenes. ${ }^{5}$ Until recently serological diagnosis has been unreliable because of cross reacting antibodies, but the present assay, only available at the Public Health Laboratory Service (PHLS) in Colindale in the UK, is more accurate and seroconversion is suggestive of disease.

Our patient had severe brain stem involvement but differed from the above descriptions in having myelitis as well. Acute demyelinating encephalomyelitis has not to our knowledge been associated with listerial infection and would be difficult to distinguish from microabscesses by imaging or evoked potentials. Partially treated meningitis and the brain stem encephalitis described in children by Bickerstaff have different clinical features. ${ }^{6}$ Other causes of a lymphocytic encephalitis were excluded.

We recommend that any child with focal brain stem and/or spinal cord signs and a cerebrospinal fluid lymphocytosis, with or without encephalitis, should be treated with high dose intravenous ampicillin and gentamicin until listerial infection has been excluded.

We thank Dr McLauclin, Central PHLS, Colindale Avenue, London for the serological assay.

1 John JF. Listeria monocytogenes. In: Harris AA, ed. Micro bial disease. Handbook of clinical neurology. Amsterdam: bial disease. Handbook of

2 Tim TW, Jackson MA, Shannon K, Cohen B, McCracken GH. Non-neonatal infection due to Listeria monocytogenes. Pediatr Infect Dis 1984;3:213-7.

3 Pollock SS, Pollock TM, Harrison MJG. Infection of the central nervous system by Listeria monocytogenes: a review of 54 adult and juvenile cases $Q \mathcal{F}$ Med 1984;53:331-40.

4 Goday A, Lozano F, Santamaria J, Gallart T, Tolosa E. Transient immunologic defect in a case of listeria rhombencephalitis. Arch Neurol 1987;44:666-7.

5 Zeman A, Bamford JM, Warlow CP, Mitchell RG. Listeria encephalitis with intermittent symptoms and serological diagnosis. F Neurol Neurosurg Psychiatry 1988;51:458-9.

6 Bickerstaff ER Brain stem encephalitis. Further observation on a grave syndrome with a benign prognosis. BMF 1957;i: on a grave

\section{No sensory neuropathy during pyridoxine treatment in homocystinuria}

\author{
Willink Biochemical \\ Genetics Unit, \\ Royal Manchester \\ Children's Hospital, \\ Pendlebury, Manchester \\ M27 1HA \\ C Mpofu \\ C Whitehouse \\ B Fowler \\ J E Wraith \\ Department of \\ Neurophysiology, \\ Royal Manchester \\ Children's Hospital \\ S M Alani \\ Correspondence to: \\ DrWraith.
}

Accepted 26 June 1991

(ArchDisChild 1991;66:1081-2)

\section{Mpofu, S M Alani, C Whitehouse, B Fowler, J E Wraith}

\begin{abstract}
Seventeen patients with cystathionine synthase deficiency homocystinuria were examined clinically and neurophysiologically for evidence of sensory neuropathy. All had received high dose pyridoxine (vitamin $\mathrm{B}-6$ ) for many years. Absence of neurological disturbance in all cases suggests long term treatment with pyridoxine in the dosages used in homocystinuric patients is not harmful.
\end{abstract}

Homcystinuria due to cystathionine synthase deficiency, an autosomal recessive disorder of methionine metabolism, results in mental, ocular, skeletal, and vascular disease if untreated. About half of the patients respond to treatment with pharmacological doses of pyridoxine (vitamin B-6). ${ }^{1}$ In our experience unresponsive patients can be managed effectively with a diet low in methionine. We have alsogiven pyridoxine to patients with no clear response to the vitamin on the basis that the exact mechanism of pyridoxine responsiveness is unknown and the assessment of partial responsiveness is difficult. High dose pyridoxine is reported to be associated with sensory neuropathy. ${ }^{2}$ Reports of toxicity in humans on relatively low pharmacological dosages $(200 \mathrm{mg} / \text { day })^{3}$ have alerted us to the possibility of pyridoxine toxicity in patients with homocystinuria.

Seventeen patients with homocystinuria (eight clearly pyridoxine responsive) who had been treated with large doses $(200-600 \mathrm{mg} /$ day $)$ of pyridoxine over many years (10-24 years) were examined clinically and neurophysiologically for evidence of neuropathy.

\section{Patients and methods}

All patients were under the care of the Willink Biochemical Genetics Unit. Sex, age at diagnosis, pyridoxine responsiveness, daily dosage, and duration of treatment are shown in the table.

Inquiry was made about sensory symptoms, including difficulty handling objects, absence of. sensation leading to unnoticed injury, weakness, and problems with balance and walking. Physical examination looked specifically at touch (cotton wool), pain (pin prick), proprioception, Romberg's sign, tendon reflexes, and observation of gait.

Plasma concentrations of pyridoxal phosphate were measured by high performance liquid chromatography. ${ }^{4}$

Electrophysiological studies were performed to look for possible subclinical effects of pyridoxine on the peripheral nerves. A supramaximal stimulation of $0.1 \mathrm{~ms}$ duration was delivered at 1 second intervals, using surface electrodes (ring electrodes for finger stimulation). The median (index finger to wrist) and sural (calf to lateral malleolus) sensory potentials were recorded using surface electrodes. Onset to latency $(\mathrm{ms})$, peak to peak amplitudes $(\mathrm{uV})$, and sensory conduction velocity $(\mathrm{m} / \mathrm{s})$ were measured. The median and lateral popliteal motor responses were recorded over abductor 